\title{
Sharing Collections of Historical Maps Online
}

\author{
Aileen R. Buckley ${ }^{\mathrm{a},}$ * \\ a Esri,Inc.,abuckley@esri.com \\ * Corresponding author
}

Keywords: Historical map, ArcGIS, Web app, Georeference, Scan, Mosaic dataset, Image Service

\begin{abstract}
:
Today's expectations for historical maps are widely different from what they were ten or even five years ago. Today, maps are expected to be freely available online and viewed in easy-to-use, interactive web apps. Collections of maps, such as maps in atlases or in a map series, are no different. While there have been great strides in developing methods for scanning and sharing smaller collections of maps online, less effort has been directed toward sharing larger and more varied collections of historical maps. Even less energy has been focused on the development of common workflows and off-the-shelf resources that could be used by many who want to digitize and share their own collections.

These deficiencies are complicated by the fact that different types of map collections require different solutions. There is wide variety in the types of map collections, including those with many maps of the same extent and map scale but varying themes (as in a thematic atlas), those with maps of varying scales and extents but a single theme (as in a topographic map series), and those with combinations of both. The workflows to convert the maps in these collections to digital format that can then be shred online must assure expediency and accuracy in the processing of the maps, despite their variations. For example, the workflow in figure 1 could be used for collections of maps with varying scales, extents, and themes.
\end{abstract}

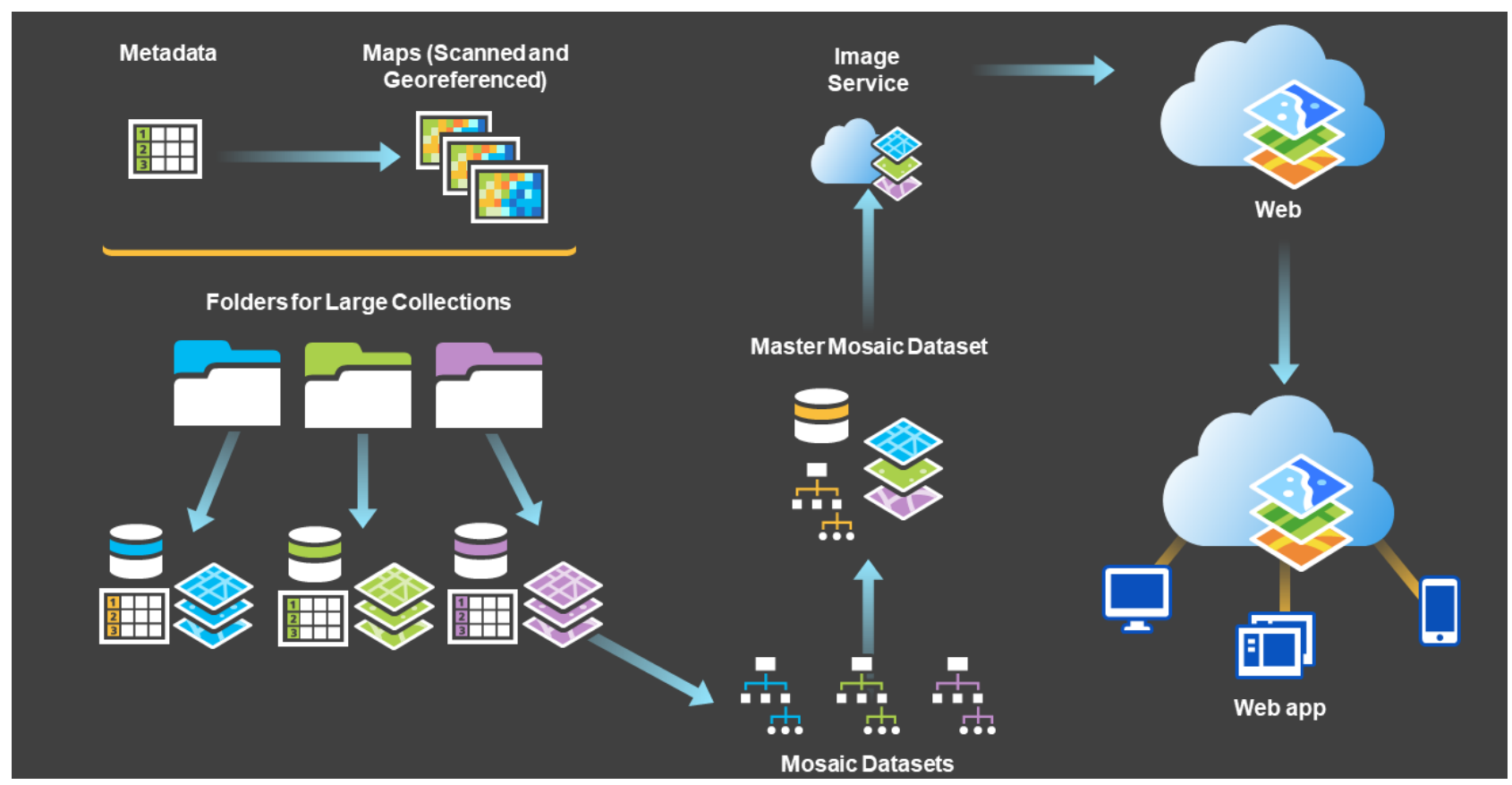

Figure 1. An ArcGIS workflow to convert historical paper maps to a format that can be viewed online in web app.

Another requirement for sharing historical map collections online is the ability to add new images or replace faulty images in the collection. The workflow in figure 1 allows for this by first updating the metadata, then adding new images to the mosaic dataset, and finally updating the image service. The view of the collection in the web app is updated automatically so no edits need be made to the app code.

The web apps used to display the map collections must also vary in order to provide an optimal experience for viewers to interact with the collection. The app in figure 2 was developed to allow viewers to explore a very large set (over 186,000) of historical topographic maps of the United States. This collection includes maps of varying scales, dates, and sizes. The app allows viewers to find maps, compare maps using transparency sliders, download maps images that can 
be used in software applications such as ArcGIS, and share the current map view with others via social media or a hyperlink.

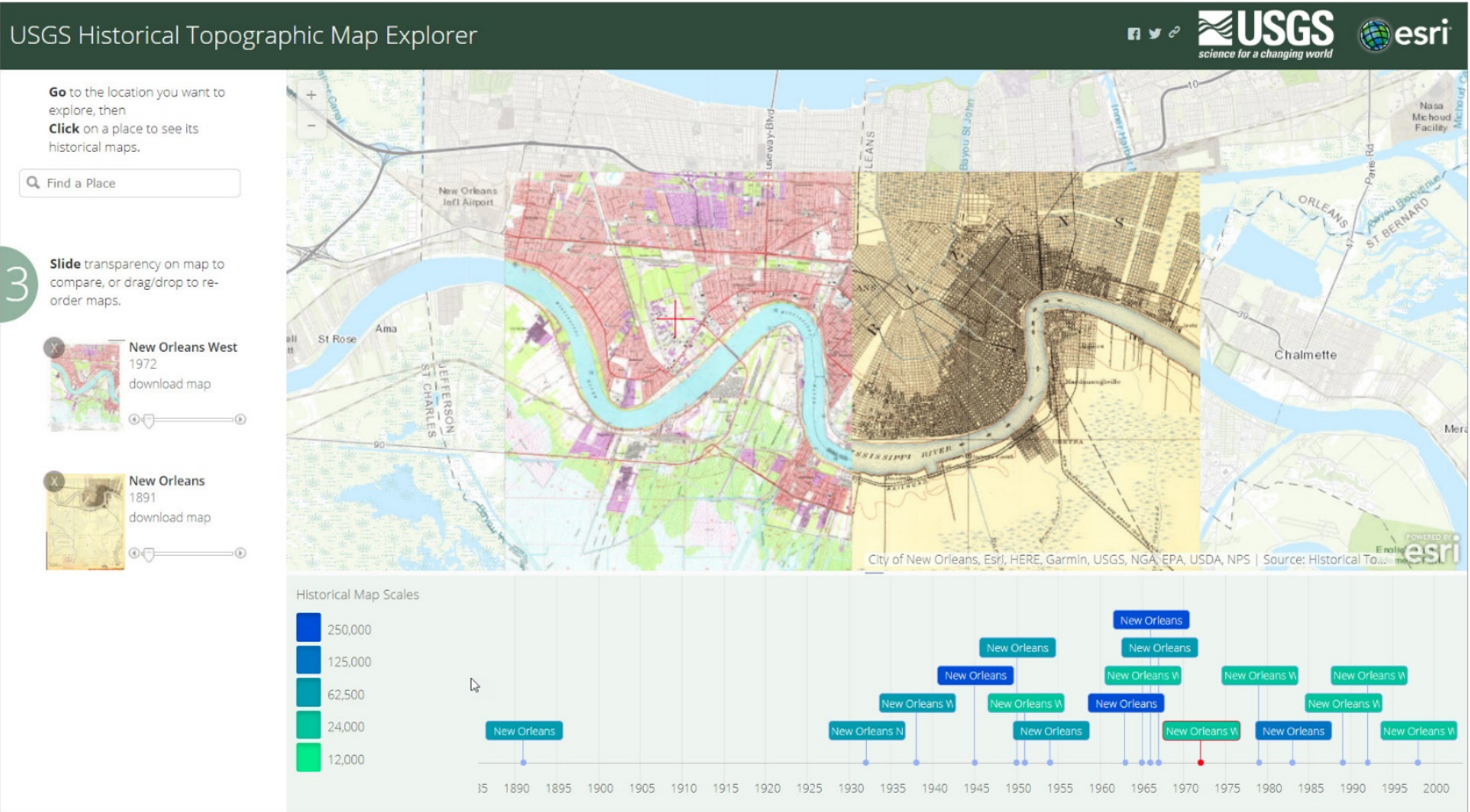

Figure 2. The USGS Historical Topographic Map Explorer.

Variations of this app could be used for other types of collections. We have explored modifications to support viewing more than 500 maps compiled by the U.S. Department of Defence during the Vietnam conflict (figure 3). The Vietnam map collection requires a slightly different app solution because there is no significant variation in the dates of the maps, although there are two map scales (1:50,000 and 1:250,000). Additionally, the marginalia on the maps contains information critical to reading the maps, such as the legend and a glossary of Vietnamese names.
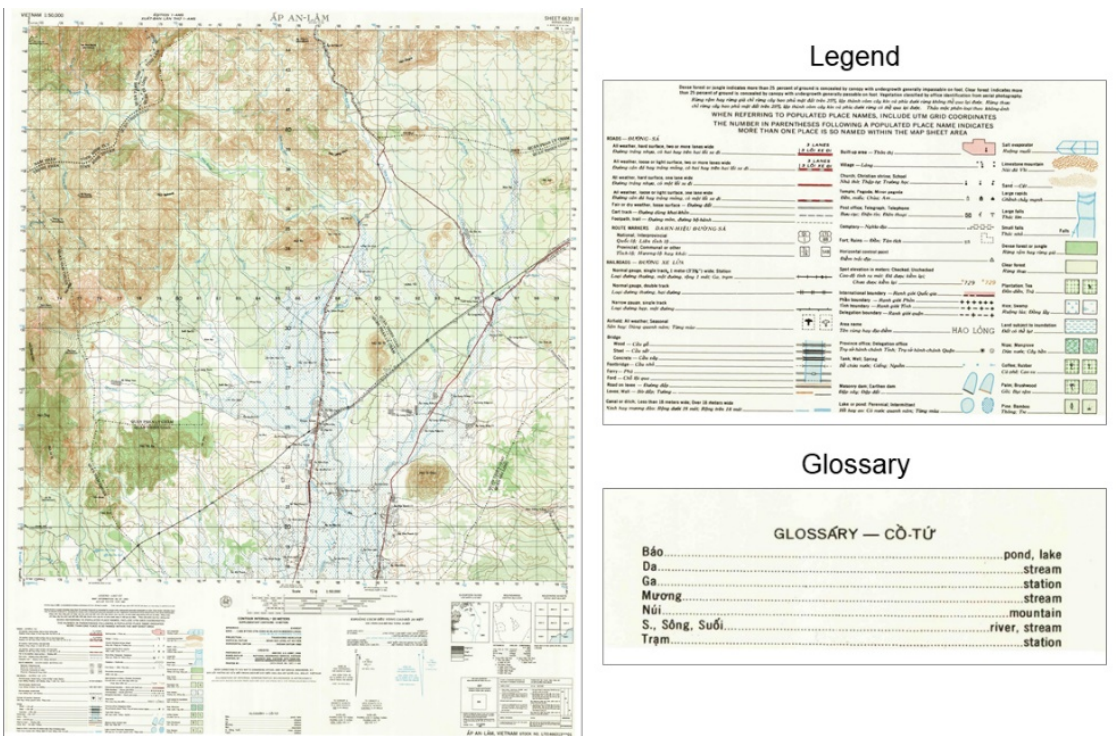

Figure 3. Example of a 1:50,000 scale map of Vietnam.

Our work to date provides solutions to sharing large collections of maps in a series or from atlases, even if the maps have different scales, dates, sizes, and themes. These collections can be shared via web apps that provide viewers with useful interactivity and functionality. We have also developed the means to update collections on a regular basis-for example, the USGS topographic map collection is now being updated quarterly. To support developers of these collections, we provide documentation on the workflow, share example datasets to allow them to test the methodology, and allow access to the web app which can be configured to conform to users' requirements. In this presentation, we detail the workflows and resources we have developed, and we demonstrate solutions for map collections of different types. 